\title{
Appearance of Sign Reversal in Geophysical Transient Electromagnetics With a SQUID Due to Stacking
}

\author{
Hans-Joachim Krause, Grigory I. Panaitov, Yi Zhang, and Marcel Bick
}

\begin{abstract}
In geophysical transient electromagnetics (TEM) measurements with HTS SQUID magnetometers, a so-called 'frequency dependence' of the stacked time transients on the repetition frequency of the transmitter, and the appearance of so-called 'sign reversals', the crossover of the stacked time transients to negative values, have been observed frequently. Recently, we have shown that both of these effects can be attributed to the summing of remnant responses from earlier transmitted pulses of the repetitive transmitter waveform [1], [2]. Although the step function inductive response for many TEM targets decays monotonically and is positive at all times, instances of sign reversal do occur. We postulate that this sign reversal is due to the typical bipolar waveform of the TEM transmitter and the stacking procedure. In this contribution, we systematically extend our analysis to binary and ternary power-law expressions for the step function response, modeling measured responses for typical ground structures. The conditions are determined under which sign reversals appear. It is shown that the effect occurs mainly in the case where a shallow slope response is followed by a rapidly decaying response at late times. Such a signal is typically measured on a resistive overburden over a conducting medium. As an example, data are presented from a location where a sign reversal was measured with a SQUID whereas none was found in the coil data. A deconvolution procedure for determining the single pulse response from measured SQUID data is proposed.
\end{abstract}

Index Terms-Geophysical inverse problems, geophysical signal processing, SQUIDs, transient electromagnetics.

\section{INTRODUCTION}

$\mathbf{I}$ $\mathrm{N}$ TIME domain transient electromagnetic (TEM) exploration, a magnetometer sensor like the SQUID has significant advantages over the commonly used induction coil [3], [4]: magnetometric TEM allows greater exploration depths and shorter acquisition times due to less stringent dynamic range requirements, and is much easier to invert using apparent resistivity analysis [5]. In the past decade, several HTS SQUID magnetometer systems for ground-based and airborne geophysical TEM exploration have been developed and their reliability has been proven in numerous field trials [4], [6]-[9]. Two peculiarities show up rather frequently in SQUID TEM recordings in the in-loop configuration [4], albeit they are not observed in corresponding induction coil data: the 'frequency dependence' of the stacked time transients on the repetition frequency of the

Manuscript received October 5, 2004

H.-J. Krause, G. I. Panaitov, and Y. Zhang are with Forschungszentrum Jülich, Juelich 52425, Germany (e-mail: h.-j.krause@fz-juelich.de).

M. Bick is with CSIRO Telecommunication and Industrial Physics, Lindfield NSW 2070, Australia (e-mail: marcel.bick@csiro.au).

Digital Object Identifier 10.1109/TASC.2005.850035 transmitter, and the appearance of so-called "sign reversals," the crossover of the stacked time transients to negative values. Similar observations may appear due to induced polarization (IP) effects or as a consequence of drift, either of magnetometer output or of the Earth's field. Recently, we have shown that both effects can be attributed to the stacking procedure performed by TEM transmitters, i.e., the summing of remnant responses from earlier transmitted pulses of the repetitive bipolar excitation waveform [1], [2], [10]. The more pronounced these artifacts are, the steeper the measured signals decay with time, so they are barely observable in coil TEM and therefore have been disregarded in the past.

Since our work stimulated a brisk discussion [2] and since SQUID TEM exploration is becoming increasingly popular [11], we attempt to cover the question of magnetometric TEM data interpretation somewhat more comprehensively in this work, extend our analysis to more realistic analytical expressions, and suggest a deconvolution procedure for determining the single pulse response from measured magnetometric TEM data.

\section{Mathematical ANALYsis of TEM Stacking}

\section{A. Expression for the Stacking Signal}

Due to the typical bipolar transmitter waveform used for TEM measurement depicted in Fig. 1, the measured response of $N$ stacked transients consists of an algebraic average of the following terms: the first measurement, containing the rising slope $\mathrm{G}$ and the falling slope $\mathrm{H}$ (time-shifted by $\mathrm{T}$ and with negative weight), plus the second measurement, containing the slopes E, F, G, H with signs $(+,-,-,+)$, plus the third with slopes $\mathrm{C}, \ldots, \mathrm{H}$ in a $(+,-,-,+,+,-)$ fashion, and so on. Late-time responses are taken into account with successively reduced weight. The stacked response obtained from $N$ averages is given by

$$
S_{T}(t)=\sum_{i=0}^{N-1}(-1)^{i} \frac{N-i}{N}[B(t+2 i T)-B(t+(2 i+1) T)]
$$

\section{B. Definition of a Versatile Analytical Expression}

To calculate the effect of remnant responses, it is necessary to adopt an analytical expression of the response transient to a single TEM step function pulse, $B(t)$. For explaining the sign reversal in [1], we used simple analytical expressions incorporating only two power law terms for the single pulse response 


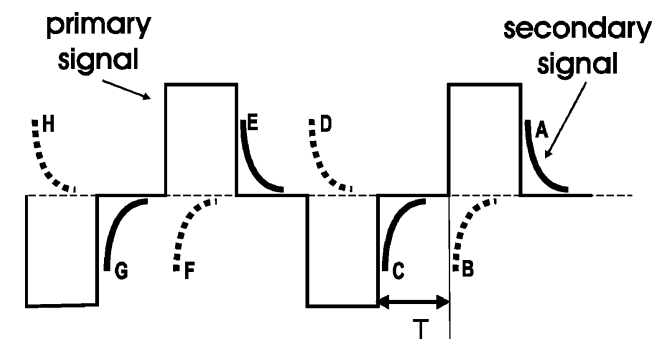

Fig. 1. Secondary field TEM traces $A, B, \ldots, H$ (thick lines) induced in the ground by the square wave transmitter signal (thin line). The thick solid lines denote pulse-off responses which are measured during off-time, the dotted represent pulse-on responses which affect later measurements during off-time.

and calculated the effect of previous remnant responses by only carrying the next two terms and neglecting the subsequent terms. Spies [2] showed explicitly that this truncation of the series to three terms is not sufficient.

In order to attain an analytical expression of maximum versatility in the description of typical ground responses, a ternary power law expression is chosen, i.e., the responses in regions I, II and III fall off with time obeying power laws with exponents $-\alpha,-\beta$, and $-\gamma$, see Fig. 2 :

$$
B(t)=\frac{B_{0}}{1+\frac{t_{0}^{-\alpha}}{t^{-\alpha}+t_{1}^{\beta-\alpha} t^{-\beta}}+\frac{t_{0}^{-\alpha} t_{1}^{\alpha-\beta} t_{2}^{\beta-\gamma}}{t^{-\gamma}+t_{3}^{\delta-\gamma} t^{-\delta}}}
$$

Here, the time $t_{0}$ denotes the onset of decay and the crossover times $t_{1}, t_{2}$ and $t_{3}$ mark the transitions between the regions I, II, III and IV, cf. Fig. 2.

The expression (2) is chosen such that it converges to both the early time asymptote,

$$
B(t) \stackrel{t \rightarrow 0}{\longrightarrow} \frac{\mu_{0} I}{2 a} \equiv B_{0}
$$

and (by simply setting $\delta=-3 / 2$ ) to the late time asymptote,

$$
B(t) \stackrel{t \rightarrow \infty}{\longrightarrow} \frac{\mu_{0} I\left(\sigma_{\mathrm{IV}} \mu_{0}\right)^{\frac{3}{2 a^{2}}}}{30 \sqrt{\pi} t^{\frac{3}{2}}}
$$

as analytically derived for a homogeneous half space by Spies and Frischknecht [5].

In case of simpler responses with lesser decay regions, the analytical expression (2) may be easily simplified by omitting terms of unwanted exponents.

\section{The Appearance of Sign Reversal}

Although the step function inductive response monotonically decays and is positive for all times, sign reversal may appear due to the $(+,-,-,+,+,-,-,+\ldots)$ sequence of remnant waveforms which is characteristic for TEM transmitters. In the following, we present a rigorous derivation that negative responses may occur for binary power-law response transients for certain choice of exponents [2].

Obviously, if the first three terms of the stacking sum are almost identical, whereas the fourth and subsequent terms are small, there will be a high likelihood of sign reversal. Thus, if the first three samples, $(+,-,-)$, are taken in the slowly decaying region II (see Fig. 2), yielding a rather large negative value, whereas the remains of the sequence, $(+,+,-,-,+\ldots)$, are

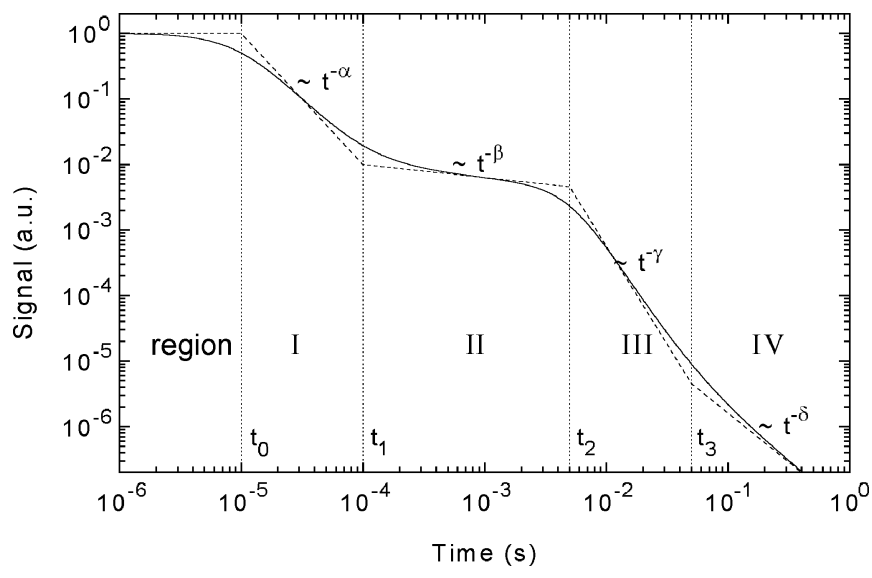

Fig. 2. Illustration of the single pulse response, with four regions, each characterized by a different power law decay. It consists of rapidly decaying early and late time regions I, III and an intermediate region II with slowly decaying response. The early-time response $\left(t<t_{0}\right)$ approaches the constant excitation field, the late time response ( $t>t_{3}$, region IV) converges asymptotically to the expected $t^{-3 / 2}$ decay. The (thick line) was modeled by (2) with the parameters $t_{0}=33, \alpha=3.5, t_{1}=0.4 \mathrm{~ms}, \beta=0.2$, $t_{2}=155 \mathrm{~ms}$, and $\gamma=5.6$.

taken in the rapidly falling region III, so that it only gives a small positive contribution in total, sign reversal is favored. Therefore, we propose that the crossover $t_{2}$ occurs between the third and the fourth remnant transient. We confine ourselves to considering the case that the single pulse transient $B(t)$ be described by

$$
B(t)=\mid \begin{array}{ll}
b \cdot t^{-\beta} & t<t_{2} \\
c \cdot t^{-\gamma} & t>t_{2}
\end{array}
$$

Then, in the limit of a large number of averages, $N$, we can express the stacking sum (1) as

$$
\begin{aligned}
B(t)= & b \cdot t^{-\beta}-b(t+T)^{-\beta}-b(t+2 T)^{-\beta} \\
& +c(t+3 T)^{-\gamma}+c(t+4 T)^{-\gamma}-c(t+5 T)^{-\gamma} \\
& -c(t+6 T)^{-\gamma}+c(t+7 T)^{-\gamma}+\ldots
\end{aligned}
$$

With the generalized Riemann Zeta Function,

$$
\zeta(z, v)=\sum_{k=0}^{\infty} \frac{1}{(k+v)^{z}}
$$

the stacked response, in the limit $N \rightarrow \infty$, may be given as

$$
\begin{aligned}
B_{(\infty)}(t)= & b T^{-\beta}\left[\left(\frac{t}{T}\right)^{-\beta}-\left(1+\frac{t}{T}\right)^{-\beta}-\left(2+\frac{t}{T}\right)^{-\beta}\right] \\
+c(4 T)^{-\gamma} & {\left[\zeta\left(\gamma, \frac{3}{4}+\frac{t}{4 T}\right)+\zeta\left(\gamma, 1+\frac{t}{4 T}\right)\right.} \\
& \left.-\zeta\left(\gamma, \frac{5}{4}+\frac{t}{4 T}\right)-\zeta\left(\gamma, \frac{3}{2}+\frac{t}{4 T}\right)\right]
\end{aligned}
$$

Without loss of generality, we may renormalize the scales

$$
b \equiv 1 \text { and } T \equiv 1
$$

and define a relative time $\tau$ and a relative crossover time $\mathrm{r}$

$$
\tau=\frac{t}{T} \quad \text { and } \quad r=\frac{t_{2}}{T}-2-\tau
$$


Here, $0<\tau<1$ and $0<\mathrm{r}<1$. Thus, determining sign reversal occurrence is reduced to finding the zeros of (8), which simplifies to

$$
\begin{aligned}
f(\beta, \gamma, \tau, r)= & \tau^{-\beta}-(1+\tau)^{-\beta}-(2+\tau)^{-\beta} \\
& +(2+\tau+r)^{\gamma-\beta} 4^{-\gamma} \\
& \times\left[\zeta\left(\gamma, \frac{3}{4}+\frac{\tau}{4}\right)+\zeta\left(\gamma, 1+\frac{\tau}{4}\right)\right. \\
& \left.\quad-\zeta\left(\gamma, \frac{5}{4}+\frac{\tau}{4}\right)-\zeta\left(\gamma, \frac{3}{2}+\frac{\tau}{4}\right)\right] \\
= & 0
\end{aligned}
$$

By numerically solving (11), we determined the relative portion of the parameter range $\mathrm{r}$ (corresponding to the crossover time $t_{2}$ between regions II and III, see Fig. 2), in which sign reversal appears. The result is displayed in Fig. 3.

From the results shown in Fig. 3, it may be deduced that sign reversal appears almost inevitably in the case of a small exponent $\beta$ in region II and a large exponent $\gamma$ in region III, provided that the repetition frequency of the TEM transmitter is chosen such that the crossover between the regions occurs between the third and the fourth remnant transient.

\section{Deconvolution Procedure for Pulse Response Recovery}

When writing down the expressions for the stacked signal for different transmitter pulse durations, $T, S_{T}(t), S_{2 T}(t), S_{4 T}(t)$ (1), one easily notices that the time-shifted contributions of the single pulse response, $B(t+i \cdot T)$, may be successively eliminated. The solution to a given 'order' (or better: to a given maximum time shift $n \cdot T$ ) is obtained by solving a set of linear equations with a triangular matrix. The result of this deconvolution, up to a period of $8 \cdot T$, is given by

$$
\begin{aligned}
& B(t)=S_{T}(t) \\
& +S_{2 T}(t+T) \\
& \left.+\frac{N-1}{N} S_{4 T}(t+2 T)+\frac{1}{N} S_{4 T}(t+3 T) \quad\right\} \text { Period } 4 T \\
& +\frac{2-N}{N} S_{8 T}(t+4 T)+\frac{2 N-3}{N} S_{8 T}(t+5 T) \\
& \left.+\frac{2 N-4}{N} S_{8 T}(t+6 T)+\frac{3-2 N}{N} S_{8 T}(t+7 T)\right\} \text { Period } 8 T \\
& +\ldots
\end{aligned}
$$

Fig. 4 depicts how the recovery of the 'true' single pulse response, $B(t)$, is increasingly improved as more terms of longer period are added to the deconvolution procedure.

Now, one might argue that it suffices to record the stacked response with a maximum pulsewidth $T$. Indeed, this is in principle true. However, a slow transmitter repetition frequency not only leads to unacceptably prolonged surveys but also has adverse effects on the measurement quality since the suppression of drift and of periodic signals like power line interference deteriorates.

\section{ANALYSIS OF SQUID TEM MEASUREMENTS}

We re-evaluated experimental data published earlier [1] which exhibited 'sign reversal'. Using the refined analytical

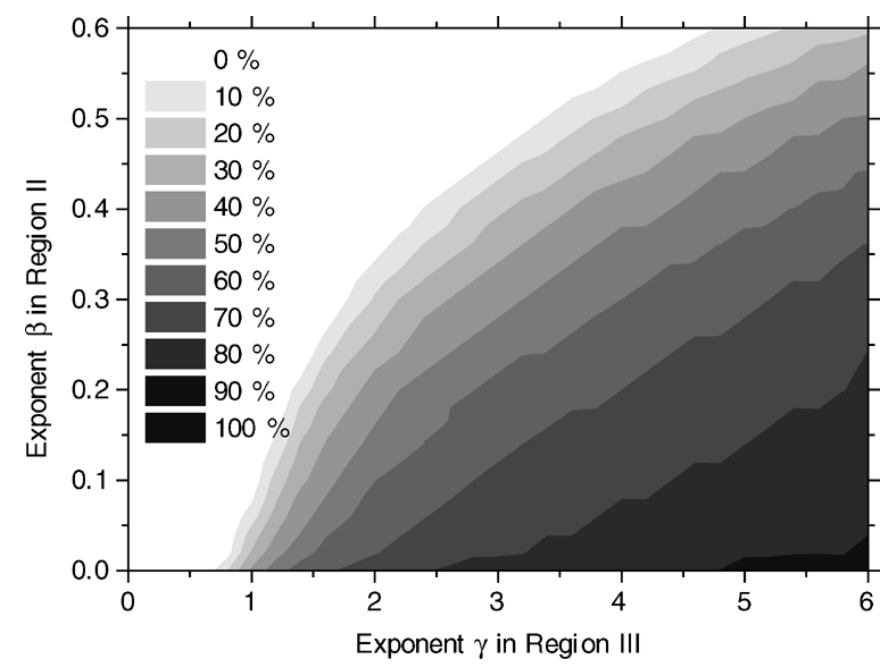

Fig. 3. Percentage of parameter range, in which sign reversal appears, for stacked magnetometric in-loop TEM in the limit of large number of averages, as a function of the exponents $\beta$ and $\gamma$ of the single pulse transient in the regions II and III, respectively (from [2], with permission).

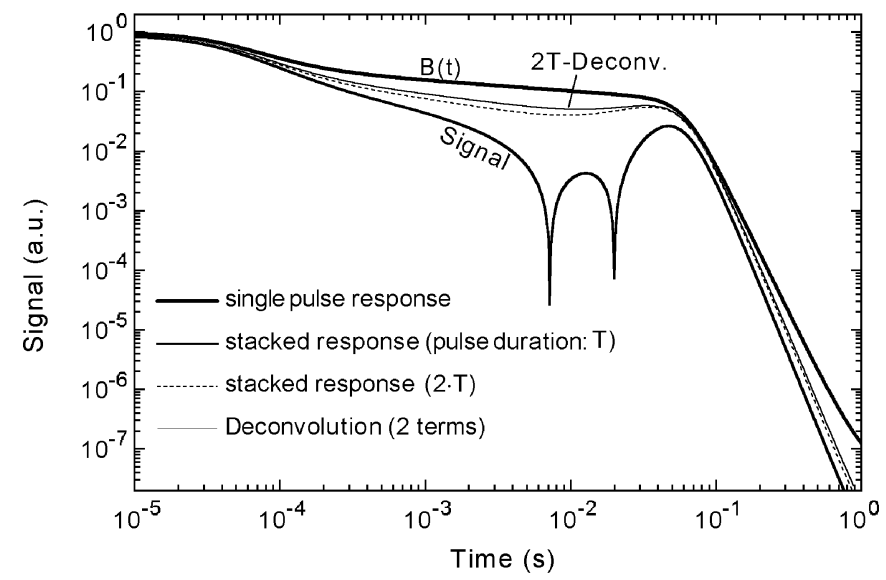

Fig. 4. Illustration of the deconvolution procedure for recovering the single pulse response (thick line) from the measured signal, obtained by stacking. For comparison: stacking with double pulse duration (dashed line).

expression (2), the single pulse response signal was modeled. Fig. 5 displays the data taken in Teveren, Germany (symbols), the adopted step function response $B(t)$ according to (2) (thin solid line), and the subsequent responses $B_{(3)}(t), B_{(4)}(t)$, $B_{(8)}(t)$ and $B_{(64)}(t)$. The latter two are indistinguishable. Consequently, it suffices to consider the first eight terms in the stacking sum (1) and to disregard the rest. We point out that our new approach not only well describes the experimental data but also exhibits 'sign reversal' even though the step function response $B(t)$ is monotonous and well-behaved.

In Fig. 6, the measured induction coil response at the same location and the differentiated $\mathrm{B}(\mathrm{t})$ curve are displayed for comparison. Both experimental and predicted coil signals exhibit no sign reversal, even though the magnetometric SQUID signal does.

Fig. 7 shows an example of SQUID and coil recordings which clearly exhibit the 'frequency dependence' effect in the SQUID data, albeit the effect is almost nonexistent in the induction coil data recorded at the same location. Again, the effect is clearly reproduced in the magnetometric data simulation using (2) with 


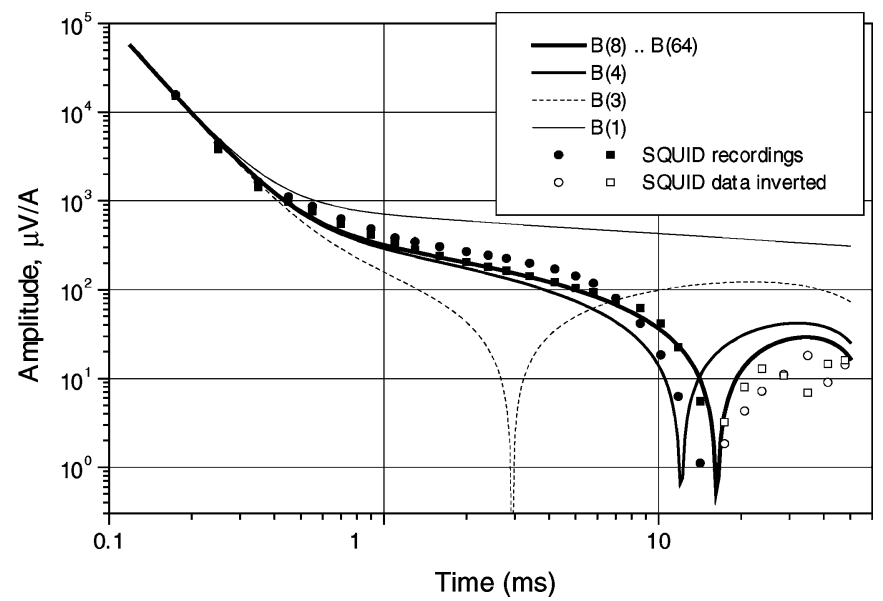

Fig. 5. Measured averaged SQUID data transient (Location: Teveren, Germany, cf. Fig. 9 of [1]). The data are modeled by the step function response $B_{(1)}$ according to (2). Parameters are $t_{0}=33, \alpha=3.5, t_{1}=0.4 \mathrm{~ms}$, $\beta=0.2, t_{2}=155 \mathrm{~ms}, \gamma=5.6, T=50 \mathrm{~ms} . B_{(N)}$ denotes the trace obtained when summing $N$ remnant transients.

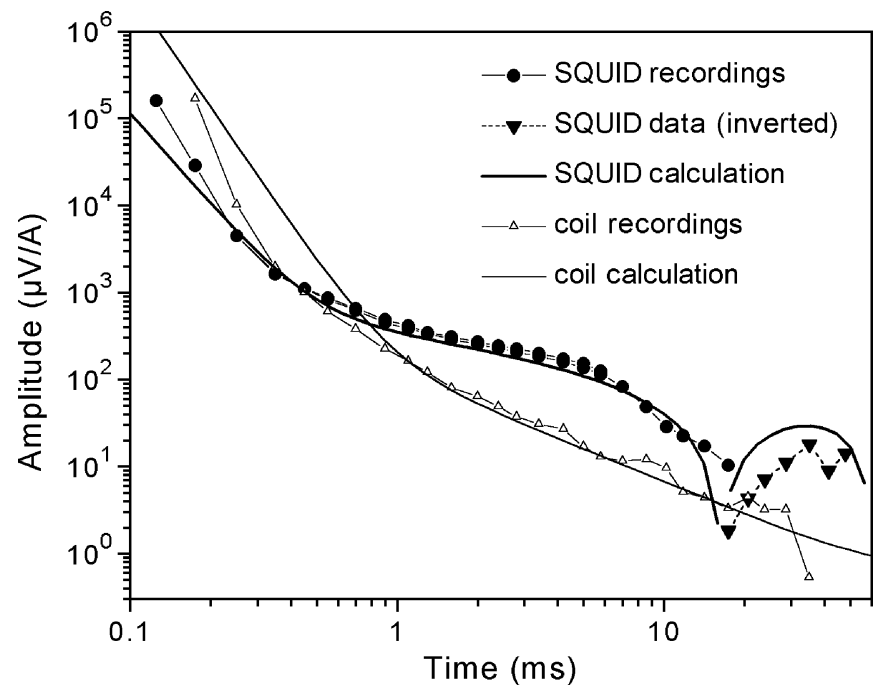

Fig. 6. SQUID (large symbols) and coil (small symbols) TEM recordings at location Teveren. Calculations of the averaged response were performed taking into account all 64 repetitive pulses. Calculation of the SQUID response show a sign inversion of the late time data (thick line), while the coil response calculated by differentiating the $\mathrm{B}(\mathrm{t})$ curve reveals no sign reversal effect (thin line).

different pulse periods $\mathrm{T}$ whereas the effect almost vanishes for the differentiated $B(t)$ curves.

\section{CONCLUSION}

By mathematical analysis, it was shown that sign reversals may appear in magnetometric vertical-component in-loop TEM. It is an artifact due to the typical bipolar waveform of the TEM transmitter and the stacking procedure. These artifacts are especially noticeable in the case where the target response is typified by an initial shallow slope followed by a rapidly decaying response at late times. Despite the step function inductive response decaying monotonically and being positive at all times, sign reversal due to the TEM stacking procedure is possible over this class of targets. Typical examples are ground structures where a resistive overburden conceals a buried conducting

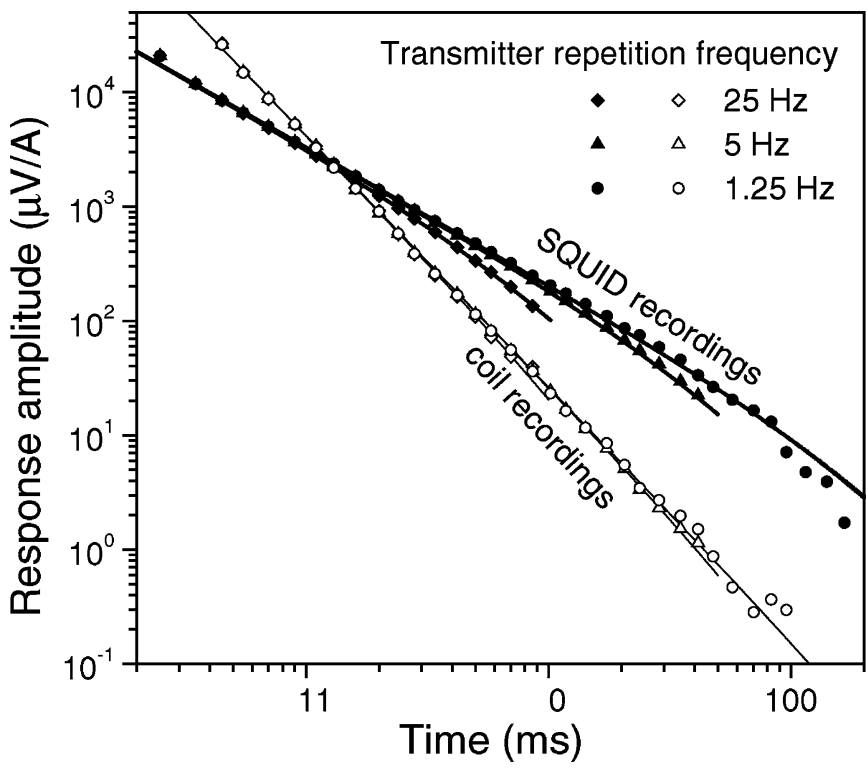

Fig. 7. Example of the frequency dependence effect. In the SQUID recordings, the effect is clearly observable for three different repetition rates $25 \mathrm{~Hz}, 5 \mathrm{~Hz}$ and $1.25 \mathrm{~Hz}$ (full symbols) and the corresponding calculated averaged earth response (thick lines), taking into account all 64 repetitive pulses. In contrast, the effect is barely visible in the corresponding coil recordings (open symbols) and differentiated calculations (thin lines).

medium. The effect is illustrated and verified by the analysis of SQUID recordings, using an analytical expression for the single pulse response. A deconvolution procedure for determining the single pulse response by measuring data with different repetition frequencies is proposed. It is expected that as remnant responses are taken into account in SQUID TEM analysis, the technique will find increased acceptance as compared to TEM surveying using induction coils.

\section{REFERENCES}

[1] G. Panaitov, M. Bick, Y. Zhang, and H.-J. Krause, "Peculiarities of SQUID magnetometer application in TEM," Geophysics, vol. 67, pp. 739-745, 2002.

[2] B. R. Spies, "Discussion on 'peculiarities of SQUID magnetometer application in TEM' by Panaitov, Geophysics 67, 739-745, 2002," Geophysics, vol. 69, pp. 624-628, 2004.

[3] C. P. Foley and K. E. Leslie, "Potential use of high Tc SQUID's for airborne electromagnetics," Explor. Geophys., vol. 29, pp. 30-34, 1998.

[4] M. Bick et al., "A HTS rf SQUID vector magnetometer for geophysical exploration methods," IEEE Trans. Appl. Supercond., vol. 9, no. 2, pp. 3780-3785, Jun. 1999.

[5] B. R. Spies and F. C. Frischknecht, "Electromagnetic sounding," in Electromagnetic Methods in Applied Geophysics, M. N. Nabighian, Ed. Oklahoma City, OK: Society of Exploration Geophysicists, 1991.

[6] C. P. Foley et al., "Field trials using HTS SQUID magnetometers for ground-based and airborne geophysical applications," IEEE Trans. Appl. Supercond., vol. 9, no. 2, pp. 3786-3792, Jun. 1999.

[7] U. Kalberkamp et al., "HTS rf-SQUID applications to geophysical exploration methods," Appl. Supercond., vol. 5, no. 7-12, pp. 205-211, 1999.

[8] V. Zakosarenko et al., "HTS dc SQUID systems for geophysical prospection," IEEE Trans. Appl. Supercond., vol. 11, no. 1, pp. 896-899, Mar. 2001.

[9] C. P. Foley et al., "Issues relating to airborne applications of HTS SQUIDs," Supercond. Sci. Technol., vol. 15, pp. 1641-1645, 2002.

[10] G. Panaitov, M. Bick, Y. Zhang, and H.-J. Krause, "Effect of repetitive transmitter signals in SQUID response in geophysical TEM," IEEE Trans. Appl. Supercond., vol. 11, no. 1, pp. 888-891, Mar. 2001.

[11] C. P. Foley, "The impact of SQUID's on Geophysics," in Proc. ISEC 2003 Conf., Sydney, Australia, Jul. 7-11, 2003. 